\title{
EDITORIAL
}

\section{COVID-19 and Pregnancy: An Unwanted Combination}

Pregnant women are not at higher risk of contracting coronavirus disease 2019 (COVID-19) and pregnant individuals with COVID-19 are more likely than nonpregnant people of similar age to require intensive care unit admission or mechanical ventilation ${ }^{1}$. COVID also leads to higher rates of death in pregnancy. Older age, high body mass index and pre-existing co-morbidities like hypertension and diabetes are associated with increased risks for more severe illness and death among pregnant people.

In this issue of journal of BCPS the paper "Maternal and Fetal Outcome of Pregnancy with COVID- 19" by AA Akhter et al. has found that $10 \%$ of the pregnant women required critical care. Neonatal death was $2 \%$ and at the same time $2 \%$ had congenital anomalies. These are few alarming data, which require further studies to confirm and update.

There is also higher risk of premature delivery and is associated with higher rates of fetal death before or during delivery. Data from the $\mathrm{CDC}$ indicate that from March 2020 to September 2021, the stillbirth rate was 273 of $21 / 653$ deliveries (1.26\%) among US women with COVID-19 compared with 7881 of $1 / 227 / 981$ deliveries (0.64\%) among women without COVID-192.

Pregnant women can be vaccinated and while there is less data available on vaccination of pregnant people ${ }^{3}$. There is no live vaccine in use, so there is no chance of developing COVID-19 from vaccination in pregnant women and fetuses. Moreover, ongoing monitoring of COVID-19 vaccination during pregnancy has shown no safety concerns so far and no adverse effects on fetal or postnatal development. COVID-19 vaccination is recommended before pregnancy and any time during pregnancy ${ }^{4}$. And any of the four currently authorized COVID-19 vaccines may be given to people who are pregnant.

Vaccinated people have been found to have antibodies against severe acute respiratory syndrome coronavirus type-2 (SARS-CoV-2) in their breast milk, which may help protect infants from infection ${ }^{5}$. Transmission of active SARS-CoV-2 virus through breast milk and breastfeeding has not been detected to date, so there is no reason to stop or avoid breast feeding.
COVID-19 vaccination is recommended for nursing mothers. The American College of Obstetricians and Gynecologists recommends that pregnant people should receive a booster dose of COVID-19 vaccine also $^{6}$.

As we have passed almost two years with COVID, it is now obvious that we have to live with it for next many years. The personal protective measures are the best weapon for self-defense which has to be supported by vaccination along with booster doses. Pregnancy increases the risk of severe disease many-fold and warrants extra-precaution from the outset.

(J Bangladesh Coll Phys Surg 2022; 40: 3)

DOI: https://doi.org/10.3329/jbcps.v40i1.57051

\section{Prof Brig Gen (retd) Mamun Mostafi}

Head, Department of Medicine \&Nephrology

GSV Medical College and GN Hospital, Dhaka, Bangladesh.

Email:mamunmostafi@yahoo.com

\section{References}

1. Wastnedge EAN, Reynolds RM, van Boeckel SR, Stock SJ, Denison FC, Maybin JA, et al. Pregnancy and COVID-19. Physiol Rev. 2021 Jan 1; 101(1): 303-18.

2. CDC: Risk of Stillbirth Is Higher Among Pregnant People With COVID-19 https://www.verywellhealth.com/cdccovid-study-pregnancy-5211825? (Accessed Dec 28, 2021)

3. https://www.rcog.org.uk/en/guidelines-research-services/ coronavirus-covid-19-pregnancy-and-womens-health/ covid-19-vaccines-and-pregnancy/covid-19-vaccinespregnancy-and-breastfeeding/ (Accessed Dec 28, 2021)

4. Magnus MC, Gjessing HK, Eide HN, Wilcox AJ, Fell DB, Håberg SE. Covid-19 Vaccination during Pregnancy and First-Trimester Miscarriage. N Engl J Med. Nov 18; 2021; 385:2008-10.

5. Breast milk can contain COVID antibodies - good news for babies https://theconversation.com/breast-milk-cancontain-covid-antibodies-good-news-for-babies-169231? (Accessed Dec 28, 2021)

6. COVID-19 Vaccination Considerations for ObstetricGynecologic Care. https://www.acog.org/clinical/clinicalguidance/practice-advisory/articles/2020/12/covid-19vaccination-considerations-for-obstetric-gynecologic-care (Accessed Dec 28, 2021) 